\title{
The effects of 8 week concurrent training on necdin and insulin levels in obese middle-aged men
}

\begin{abstract}
Obesity per se is a major global health problem leading to an increase in morbidity and premature mortality however increasing physical activity often results in a marked weight loss. Necdin has been shown to be an important negative regulator of adipogenesis of the white adipose tissue. We hypothesized that exercise training would impact necdin synthesis inhibiting adipocity and ultimately reducing obesity. Thus, this study was conducted to determine the effects of 8weeks concurrent training on plasma necdin levels in obese middle-aged men. Twenty three sedentary obese middle-aged men were randomly assigned to concurrent training group (CTG: $n=12$ ) or control group (CG: $n=11$ ). The subjects in concurrent training group performed endurance and resistance training on the same day (3days a week, $60 \mathrm{~min}$ a session each day) for 8 weeks. At the end of the study, there were significant decreases in total body weight (up to $3.5 \mathrm{~kg}$ ), body mass index (BMI; $1.2 \mathrm{~kg}$ / $\mathrm{m}^{2}$ ), waist hip ratio (WHR; 0.02 unit) and the fasting plasma insulin levels (up to $58 \%$ ) and insulin resistance (HOMA-IR; $\mathrm{P}<0.05$ ) in $\mathrm{CTG}$ group compared with the CG group. However, there were no significant differences in the fasting plasma levels of glucose or necdin between the two groups at the end of the study. Concurrent training is a viable tool in reducing body weight. There seems to be no association between 8 weeks concurrent training-induced weight loss and the total plasma necdin levels in obese middle-aged men.
\end{abstract}

\section{Summary statement}

Obesity is a major health problem affecting over $40 \%$ of the world population. Increase in physical activity helps weight loss. In this study, we have demonstrated that nedcin, a potential endogenous anti-obesity protein, has no role in exercise-induced weight loss.

Keywords: concurrent training, necdin, adipocyte differentiation, obesity, insulin resistance

\author{
Volume 4 Issue I - 2017 \\ Mehrzad Moghadasi,' Javad Mehrabani,'2 \\ Zahra Momennasab, ${ }^{3}$ Ebrahim K Naderali ${ }^{4}$ \\ 'Department of Exercise Physiology, Islamic Azad University, Iran \\ ${ }^{2}$ Department of Exercise Physiology, University of Guilan, Iran \\ ${ }^{3}$ Department of Exercise physiology, Islamic Azad University, Iran \\ ${ }^{4}$ Liverpool Hope University, UK
}

Correspondence: Ebrahim K Naderali, Faculty of Health Sciences, Liverpool Hope University, Hope Park Campus, Liverpool, LI6 9JD, UK, Tel +44 015 I29I 3439, Fax +44 015 1291 3414,Email naderae@hope.ac.uk

Received: January 17, 2017| Published: March 03, 2017
Abbreviations: CC, control group; CTG, concurrent training group; BMI, body mass index; WHR, waist hip ratio; WAT, white adipose tissue; GLP, glucagon-like peptide-1; GIP, gastric inhibitory polypeptide; CCK, cholecystokinin

\section{Introduction}

Obesity is now a major global health problem contributing to significant premature morbidity and mortality. It is manifestation of an increase in caloric intake and sedentary lifestyle and leads to debilitating conditions which include type 2 diabetes mellitus, cardiovascular diseases, high blood pressure, and a number of cancers. Obesity presents itself with an increase in adipocytes, primarily white adipose tissue (WAT; adipocytes) which is a highly active metabolic and endocrine organ with an important role in the energy storage and metabolism. ${ }^{1}$ White adipocytes are terminally differentiated postmitotic cells arising from progenitor cells (preadipocytes) or mesenchymal stem cells within the WAT. ${ }^{2}$ Therefore, it is likely that white adipocyte number is controlled by the rate of preadipocyte proliferation which may indicate contribution of preadipocyte proliferation to etiology of the obesity. Nonetheless, the molecular mechanism(s) regulating preadipocyte proliferation during adipose tissue development remains unclear. There are a number of hypotheses linking various endogenous proteins/molecules such as insulin, leptin, adiponectin, resistin and nedcin amongst many others to weight gain/ loss. The latter has been strongly implicated in surgical-induced weight loss process. However, there is no data on the role of nedcin in physiological-induced (exercise) weight loss.

Necdin is encoded by the NDN gene in humans. ${ }^{3}$ It was originally identified as a gene product induced in neurally differentiated embryonal carcinoma stem cells. ${ }^{4}$ A number of studies have demonstrated that postmitotic cells such as neurons and skeletal myocytes are the main sources of necdin gene expression. ${ }^{5,6}$ Expression of the necdin gene is controlled through genomic imprinting, a placental mammalspecific epigenetic mechanism. ${ }^{7,8}$ Necdin has been implemented in complement retinoblastoma protein to prevent postmitotic neurons cell proliferation, ${ }^{9}$ interactions with viral oncoproteins and cellular E2F family proteins ${ }^{10,11}$ and with contribution to apoptosis suppression and inhibiting p53-dependent apoptosis. ${ }^{12}$ These reports suggest that necdin serves as an anti- cell proliferation and anti-apoptotic protein in postmitotic cells. ${ }^{13}$

Exercise training is a useful strategy for decreasing adipocity and thus reducing obesity; ${ }^{14}$ however the exact mechanism(s) involved in the beneficial effects of exercise training on obesity has not been fully elucidated. We hypothesized that exercise training would alter plasma necdin levels leading to reduction in adiposity.

\section{Materials and methods}

\section{Subjects}

Twenty three sedentary obese middle-aged men with the mean $( \pm \mathrm{SD})$ body mass index of $32.9 \pm 2.4 \mathrm{~kg} / \mathrm{m}^{2}$, volunteered to participate 
in 8 weeks concurrent exercise regimen study. All subjects were asked to complete a personal health and medical history questionnaire, which served as a screening tool. All subjects had a very sedentary lifestyle and were considered to have an inactive lifestyle for at least 6 months before their enrolment into the study. The participants were non-smokers and free from unstable chronic conditions including dementia, unstable angina and retinal haemorrhage or detachment. Furthermore subjects had no history of myocardial infarction (MI), stroke, cancer, dialysis, restraining orthopaedic or neuromuscular diseases. Informed consent was obtained from all subjects prior to the study commencement. The study was approved by the Marvdasht branch of the Islamic Azad University Ethics Committee. The subjects were randomly assigned to one of the concurrent training group (CTG: $\mathrm{n}=12$ ) or control group (CG: $\mathrm{n}=11$ ).

\section{Exercise protocol}

The concurrent training group performed $30 \mathrm{~min}$ endurance exercise at an intensity corresponding to $70-85 \%$ individual maximum heart rate followed by a circuit weight training schedule. The resistance training was circularly performed in 8 stations and included 2-4 sets with 8-12 maximal repetitions at $65-80 \%$ of $1-\mathrm{RM}$ in each station. Each circuit and set was separated by $2-3 \mathrm{~min}$ and $30 \mathrm{~s}$ rest, respectively. The intervention was performed 3days a week for 8 weeks.

\section{Anthropometric and body composition measurements}

Height was measured with a fixed stadiometer (Seca, Germany) and weight was measured with a regularly calibrated electronic scale (Seca, Germany). These data was used to determine body mass index (BMI) for each participant. The waist circumference was determined by obtaining the minimum circumference (narrowest part of the torso, above the umbilicus) and the maximum hip circumference while standing with their heels together. The waist to hip ratio (WHR) was calculated by dividing waist by hip circumference $(\mathrm{cm})$.

\section{Blood sampling and analyses}

Fasting blood samples $(10 \mathrm{ml})$ were collected from the antecubital Tablel Anthropometric characteristics (mean \pm SD) of the subjects before and after the training

\begin{tabular}{|c|c|c|c|c|}
\hline & \multicolumn{2}{|c|}{ Control(mean $\pm S D)$} & \multicolumn{2}{|c|}{ Concurrent Training(mean $\pm S D)$} \\
\hline & Pre training & Post training & Pre training & Post training \\
\hline Body Weight(Kg) & $106.6 \pm 4.7$ & $106.5 \pm 7.6$ & $98.08 \pm 6.9$ & $94.5 \pm 7.4 *+$ \\
\hline $\mathrm{BMI}\left(\mathrm{Kg} / \mathrm{m}^{2}\right)$ & $34 . I \pm 2.5$ & $34.1 \pm 3.3$ & $31.8 \pm 1.7$ & $30.6 \pm 1.4^{*} \dagger$ \\
\hline WHR & $0.95 \pm 0.03$ & $0.95 \pm 0.04$ & $0.96 \pm 0.04$ & $0.94 \pm 0.04 * \dagger$ \\
\hline
\end{tabular}

$* \mathrm{P}<0.05$ for between-group differences, ${ }^{\mathrm{t}} \mathrm{P}<0.05$, pre training vs. post training values

Table 2 Biochemical characteristics (mean $\pm S D$ ) of the subjects before and after the training

\begin{tabular}{|c|c|c|c|c|}
\hline & \multicolumn{2}{|c|}{ Control(mean $\pm S D)$} & \multicolumn{2}{|c|}{ Concurrent training(mean $\pm S D)$} \\
\hline & Pre training & Post training & Pre training & Post training \\
\hline Fasting Glucose(mmol/l) & $5.6 \pm 0.4$ & $5.6 \pm 0.6$ & $5.2 \pm 0.7$ & $5.4 \pm 0.8$ \\
\hline Fasting Insulin & $12.7 \pm 6.7$ & $14.3 \pm 8.4$ & $11.4 \pm 8.9$ & $6.8 \pm 6.2 * \dagger$ \\
\hline HOMA-IR & $3.2 \pm 1.9$ & $3.5 \pm 2.0$ & $2.7 \pm 2.3$ & $1.7 \pm 1.6 * \dagger$ \\
\hline $\operatorname{Necdin}(p g / m l)$ & $311.9 \pm 260.4$ & $281.6 \pm 221.6$ & $324.1 \pm 207.5$ & $234.4 \pm 146.3 \dagger$ \\
\hline
\end{tabular}

$* \mathrm{P}<0.05$ for between-group differences, ${ }^{\dagger} \mathrm{P}<0.05$, pre training $v s$. post training values

Citation: Moghadasi M, Mehrabani J, Momennasab Z, et al.The effects of 8 week concurrent training on necdin and insulin levels in obese middle-aged men. J Diabetes Metab Disord Control. 20I7;4(I):26-29. DOI: I0.15406/jdmdc.2017.04.00I0I vein at the same time on day 1 and at the end of the study (week 8). The serum was frozen at $-80^{\circ} \mathrm{C}$ for subsequent laboratory analysis. The plasma necdin levels were measured using an enzymelinked immunosorbent assay (ELISA) kits with sensitivity of $24 \mathrm{pg} /$ $\mathrm{ml}$ (BioCEP, China). Plasma glucose levels were determined by an enzymatic (GOD-PAP, Glucose Oxidase-Amino Antipyrine) colorimetric method (Pars Azmoun, Tehran, Iran). The intra- and inter-assay coefficients of variation for glucose were $<1.3 \%$ with a sensitivity of $1 \mathrm{mg} / \mathrm{dl}$. The plasma insulin levels were measured by a radioimmunoassay (RIA) and the insulin resistance index determined by HOMA-IR was calculated according to the homeostasis model assessment. ${ }^{15}$

\section{Statistical analysis}

Descriptive statistics were computed and distributions of all variables were assessed for normality. Paired 't-test' was used to compute mean $( \pm \mathrm{SD})$ changes in the variables in control and concurrent training group pre and after the intervention. Differences among groups were assessed by using independent- samples 't-test'. The level of significance in all statistical analyses was set at $\mathrm{P}<0.05$. The statistical software program SPSS.19 was used for all data analysis.

\section{Results}

The physical characteristics of the subjects are presented in Table 1. Before the intervention, there were no significant differences in the body weight, BMI and WHR among the groups. At the end of the study, there were significant decreases in the body weight (up to $3.5 \mathrm{~kg}$ ), BMI (up to $1.2 \mathrm{~kg} / \mathrm{m}^{2}$ ) and WHR (0.02 unit) (for all, $\mathrm{P}<0.05$ ) in concurrent training group compared to their control group counterparts. The results also showed that fasting insulin (up to $58 \%$ ) and insulin resistance index determined by HOMA-IR were significantly decreased (for all, $\mathrm{P}<0.05$ ) in $\mathrm{CTG}$ group compared to $C G$ group. However, there were no significant differences in the plasma necdin and fasting glucose levels between the two groups at the end of the study (Table 2). 


\section{Discussion}

The prevalence of obesity, especially central obesity, has increased dramatically in recent years. Obesity causes numerous health problems including type 2 diabetes mellitus, cardiovascular diseases, high blood pressure, and have been implicated in a number of malignancies. ${ }^{16}$ Moreover, obesity per se as comorbidity increases morbidity and financial cost associated with patient care. ${ }^{17}$ Currently there are no effective means of managing obesity however change in lifestyle (reduced caloric intake together with increased physical activities) has been shown as an effective way of managing obesity. That being said that the mechanism(s) of exercise-induced weight loss is not fully understood as yet.

Obesity is the manifestation of an increase in the volume and/or the number of adipocytes. The obese individuals have greater number of adipocytes than the lean individuals. ${ }^{18}$ In fact increase in lipid depots in white adipocytes and adipocyte differentiation has been thought to be the major causes of fat mass expansion. ${ }^{18,19}$ Furthermore, the mechanisms of fat tissue growth during the evolution of obesity vary among depots- adipocyte number increases to different levels in certain depots after excessive food intake. ${ }^{19}$ Thus, identifying the mechanism(s) controlling preadipocyte proliferation could play an important role in determining the etiology of obesity and potentially helping us to devise an effective strategy to prevent obesity-associated morbidity and premature mortality.

There are numerous pre-clinical and clinical studies reporting the importance of lifestyle changes that include diet and exercise in tackling obesity. ${ }^{20,22}$ Necdin comprises 325 amino acids and is a member of the type 2 melanoma-associated antigen proteins. Necdin was originally found in neurally differentiated mouse embryonal carcinoma cells, but a wider role has been postulated via its ectopic expression, which suppresses the proliferation of several cell lines. ${ }^{23}$ In preadipocytes with differentiation defects, such as IRS-1 knockout cells, necdin levels are markedly increased. Reducing necdin levels in IRS-1 knockout preadipocytes using small interfering RNA reverses the blockade in differentiation and restores both the phenotype and gene expression profile of wild-type cells, including overexpression reduction of known suppressors of preadipocyte-adipocyte transition, preadipocyte factor- 1 and Wnt10a. ${ }^{24}$ These features indicate that necdin is not only an important negative regulator of white fat adipogenesis but also is involved at early stages in the process. Therefore we examined whether exercise will induced changes in necdin, and if so, how impactful necdin changes will be on obesity level in sedentary subjects. Our results demonstrated that although the body weight, BMI, WHR and HOMA-IR were significantly decreased $(\mathrm{P}<0.05)$ after the completion of the prescribed exercise regimen during our study period, the necdin levels remained unchanged, indicating that concurrent exercise training for 8 weeks had no effect in necdin production. This may also in turn suggest that necdin, at least in short-term (8week excerise) is not involved in adiposity regulation, primarily the weight loss. However, our hypothesis is in contrast to a recent report suggesting necdin-E2F4 interaction as one of the mediator for change in insulin sensitivity observed after Rouxen-Y gastric bypass surgery. ${ }^{25}$

This discrepancy may be due to a number of factors:

I. The onset of weight loss with surgical intervention (Roux-en-Y gastric bypass) is much faster than the one observed in our study

II. At any given time, the magnitude of the weight loss is generally greater with surgical interventions than exercise regimen and
III. Surgical intervention-induced changes in neurohormonal factors such as changes in gut hormone levels of GLP-1, GIP, Orexin, Ghrelin, CCK may have as yet unknown effects on necdin synthesis and secretion. This hypothesis merits further investigation.

Insulin receptor substrate proteins regulate necdin-E2F4 interaction that represses peroxisome-proliferator-activated receptor gamma $(\operatorname{PPAR} \gamma)$ transcription via a cyclic AMP response element binding protein dependent pathway. ${ }^{24}$ PPAR $\gamma$ has been proven crucial in the control of terminal adipocyte differentiation, and is the target for insulin-sensitizing thiazolidinediones (TZDs) which is used for treatment of type 2 diabetic mellitus. ${ }^{24}$ A previous animal study determined that the IGF-1 signalling pathway promotes adipocyte differentiation and therefore insulin sensitivity via suppression of necdin expression, which represses PPAR $\gamma$ promoter activity via interaction with E2F4. ${ }^{24}$ In contrast, our study did not show any significant relationship between necdin and body weight, BMI, WHR and HOMA-IR. However, as we did not measure IGF-1 levels in this study (one of the limitation of our study), we cannot rule out potential involvement of IGF-1 mechanism responsible for the post exercise changes in necdin levels. Therefore, further studies are required to elucidate potential impact of exercise on IGF-1 and plasma necdin levels in relation to adipocity regulation.

\section{Acknowledgments}

The authors wish to thanks all subjects who are participated in this study.

\section{Author contribution}

All authors have contributed equally on the concept, design, conduct, manuscript compilation and submission.

\section{Conflict of interest}

Author declares that there is no conflict of interest.

\section{References}

1. Kershaw EE, Flier JS. Adipose tissue as an endocrine organ. $J$ Clin Endocrinol Metab. 2004;89(6):2548-2556.

2. Fujiwara K, Hasegawa K, Ohkumo T, et al. Necdin controls proliferation of white adipocyte progenitor cells. PLoS One. 2012;7(1):e30948.

3. MacDonald HR, Wevrick R. The necdin gene is deleted in Prader-Willi syndrome and is imprinted in human and mouse. Hum Mol Genet. 1997;6(11):1873-1878.

4. Maruyama K, Usami M, Aizawa T, et al. A novel brain-specific mRNA encoding nuclear protein (necdin) expressed in neurally differentiated embryonal carcinoma cells. Biochem Biophys Res Commun. 1991;178(1):291-296.

5. Taniura H, Taniguchi N, Hara M, et al. Necdin, a postmitotic neuronspecific growth suppressor, interacts with viral transforming proteins and cellular transcription factor E2F1. J Biol Chem. 1998;273(2):720-728.

6. Kuwajima T, Taniura H, Nishimura I, et al. Necdin interacts with the Msx2 homeodomain protein via MAGE-D1 to promote myogenic differentiation of $\mathrm{C} 2 \mathrm{C} 12$ cells. The Journal of Biological Chemistry. 2004;279(39):40484-40493.

7. Jay P, Rougeulle C, Massacrier A, et al. The human necdin gene, NDN, is maternally imprinted and located in the Prader-Willi syndrome chromosomal region. Nat Genet. 1997;17(3):357-361.

8. MacDonald HR, Wevrick R. The necdin gene is deleted in Prader-Willi syndrome and is imprinted in human and mouse. Hum Mol Genet. 1997;6(11):1873-1878. 
9. Hayashi Y, Matsuyama K, Takagi K, et al. Arrest of cell growth by necdin, a nuclear protein expressed in postmitotic neurons. Biochem Biophys Res Commun. 1995;213(1):317-324.

10. Kobayashi M, Taniura H, Yoshikawa K. Ectopic expression of necdin induces differentiation of mouse neuroblastoma cells. J Biol Chem. 2002;277(44):42128-42135.

11. Kurita M, Kuwajima T, Nishimura I, et al. Necdin downregulates CDC2 expression to attenuate neuronal apoptosis. $J$ Neurosci. 2006;26(46):12003-12013.

12. Hasegawa K, Yoshikawa K. Necdin regulates p53 acetylation via Sirtuin1 to modulate DNA damage response in cortical neurons. $J$ Neurosci. 2008;28(35):8772-8784.

13. Yoshikawa K. Cell cycle regulators in neural stem cells and postmitotic neurons. Neurosci Res. 2000;37(1):1-14.

14. Mohebbi H, Rahmani Nia F, Hassan Nia S, et al. High-intensity endurance training improves adiponectin mRNA and plasma concentrations. Eur J Appl Physiol. 2012;112(4):1207-1214.

15. Matthews DR, Hosker JP, Rudenski AS, et al. Homeostasis model assessment: insulin resistance and beta-cell function from fasting plasma glucose and insulin concentrations in man. Diabetologia. $1985 ; 28(7): 412-419$

16. Kopelman PG. Obesity as a medical problem. Nature. 2000;404(6778):635-643.

17. Colmegna I, Hitchon CA, Bardales MC, et al. High rates of obesity and greater associated disability among people with rheumatoid arthritis in Canada. Clin Rheumatol. 2015;35(2):457-460.
18. Spalding KL, Arner E, Westermark PO, et al. Dynamics of fat cell turnover in humans. Nature. 2008;453(7196):783-787.

19. Tchoukalova YD, Votruba SB, Tchkonia T, et al. Regional differences in cellular mechanisms of adipose tissue gain with overfeeding. Proceedings of the National Academy of Science of the United States of America. 2010;107(42):18226-18231.

20. Naderali EK, Fatani S, Williams G. Chronic withdrawal of a highpalatable obesity-inducing diet completely reverses metabolic and vascular abnormalities associated with dietary-obesity in the rat. Atherosclerosis. 2004;172(1):63-69.

21. Fatani S, Abubakari AR, Itua I, et al. Effects of diet-induced obesity on protein expression in insulin signaling pathways of skeletal muscle in male Wistar rats. Int J Gen Med. 2012;5:573-582.

22. Sari Sarraf V, Aliasgarzadeh A, Naderali MM, et al. A combined continuous and interval aerobic training improves metabolic syndrome risk factors in men. Int J Gen Med. 2015;8:203-210.

23. Yoshikawa K. Cell cycle regulators in neural stem cells and postmitotic neurons. Neurosci Res. 2000;37(1):1-14.

24. Tseng YH, Butte AJ, Kokkotou E, et al. Prediction of preadipocyte differentiation by gene expression reveals role of insulin receptor substrates and necdin. Nat Cell Biol. 2005;7(6):601-611.

25. Pamuklar ZN, Chen J, Muehlbauer M, et al. Necdin-E2F4 interaction provides insulin-sensitizing effect after weight loss induced by gastric bypass surgery. Surg Obes Relat Dis. 2013;9(1):94-99. 